\title{
Search for R-parity violating supersymmetry with the ATLAS detector
}

\section{Paul D. Jackson, on behalf of the ATLAS Collaboration*}

SLAC National Accelerator Laboratory

E-mail: paul.jacksondcern.ch

R-parity violation in supersymmetry gives rise to many unique experimental signatures. We describe searches with the ATLAS detector for supersymmetry with R-parity violating decays. Examples include searches for resonant sneutrino decays to an electron and a muon, and displaced vertices arising from the late decays of heavy objects with a muon in the final state. The most recent results on these channels are presented based on data recorded with the ATLAS detector in $7 \mathrm{TeV} p p$ collisions at the CERN Large Hadron Collider in 2010 and 2011.

The 2011 Europhysics Conference on High Energy Physics-HEP 2011,

July 21-27, 2011

Grenoble, Rhône-Alpes France

\footnotetext{
* Speaker.
} 


\section{Introduction}

An essential aspect of all supersymmetric (SUSY) models is the R-parity quantum number $R_{p}=(-1)^{3 B+L+2 S}$, where $B$ denotes the baryon number, $L$ the lepton number and $S$ the spin of a particle. In the most general formulations of supersymmetric theory $R$-parity is not conserved, allowing couplings between two ordinary fermions and a squark $(\tilde{q})$ or slepton $(\tilde{\ell})$. The conservation of R-parity forbids strong baryon and lepton number violation simultaneously and leaves the lightest particle as a natural dark matter candidate. R-parity violating (RPV) couplings are allowed in scenarios which forbid either the baryon or lepton number violating terms. Here, the dark matter candidate is unstable due to new channels being opened.

This note reports on two searches performed by the ATLAS collaboration [1] which can be interpreted in terms of constraints on RPV SUSY [2]. We search for a resonant structure decaying, into oppositely charged electron and muon pairs. A separate analysis searches for displaced vertices, potentially arising from the decay of heavy particles with lifetimes between picoseconds and nanoseconds.

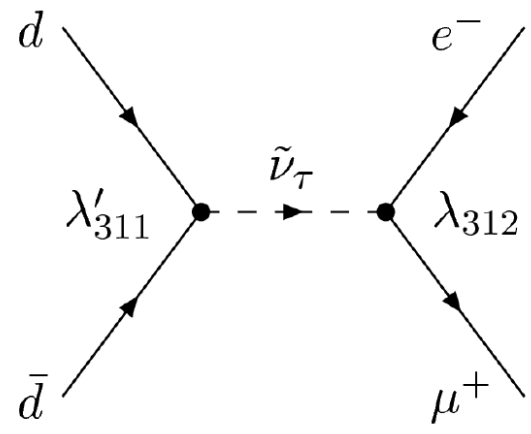

(a) $\tilde{v}_{\tau} \rightarrow e \mu$ decay

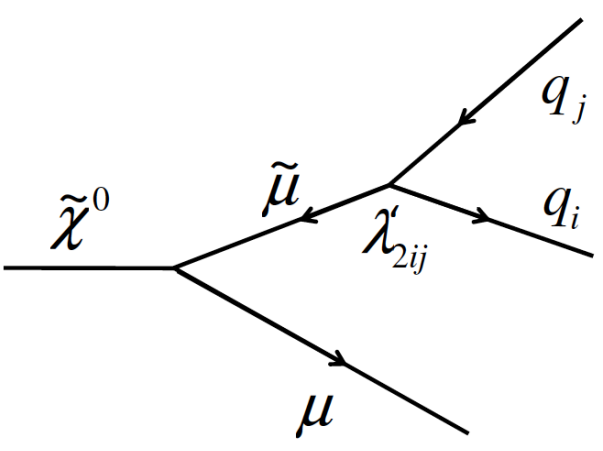

(b) $\tilde{\chi}^{0}$ decaying into a muon and two jets, via a virtual $\tilde{\mu}$, with RPV coupling $\lambda_{2 i j}^{\prime}$.

Figure 1: Feyman diagrams for the two RPV processes under consideration.

\section{2. e- $\mu$ resonance}

By fixing all RPV couplings except $\lambda_{311}^{\prime}\left(\tilde{v}_{\tau} \rightarrow d \bar{d}\right)$ and $\lambda_{312}\left(\tilde{v}_{\tau} \rightarrow e \mu\right)$ to zero, and assuming that $\tilde{v}_{\tau}$ is the lightest supersymmetric particle, the contributions to the $e \mu$ final state originate from the $\tilde{v}_{\tau}$ only [3]. To select $e \mu$ candidates, the electron is required to have transverse momentum $\left(p_{\mathrm{T}}\right)>25 \mathrm{GeV}$, to have pseudorapidity $|\eta|<1.37$ or $1.52<|\eta|<2.47$ and satisfy shower shape, track quality and matching criteria. The muon candidate must be reconstructed in both the inner detector and muon spectrometer, have $p_{\mathrm{T}}>25 \mathrm{GeV}$ and $|\eta|<2.4$, the inner detector track must be isolated from other tracks. The electron and muon must be separated by $\sqrt{\left(\Delta \eta^{2}+\Delta \phi^{2}\right)}>0.2$. The $e \mu$ candidates must be composed of exactly one electron and one muon of opposite charges.

Standard Model backgrounds to the search can arise from two categories: those with a real final state $e \mu$ pair $\left(Z / \gamma^{*} \rightarrow \tau \tau, t \bar{t}\right.$, single top, $W W, Z Z$ and $\left.W Z\right)$, and processes which give fake back- 
grounds ( $W / Z+\gamma, W / Z+$ jets and multijet events with photons or jets reconstructed as leptons). All processes listed in the first category, along with photon related backgrounds, are estimated from MC simulation (described elsewhere [4]). The remaining fake backgounds are described using a $4 \times 4$ matrix method described in [3]. The lepton definitions are loosened to allow events to be classified based on whether they pass or fail the loose and standard requirements. These can then be used to quantify the overall lepton efficiencies and jet fake backgrounds.

Analysing $1.07 \pm 0.04 \mathrm{fb}^{-1}$ of data collected in 2011 using single lepton triggers (measured to be $100 \%$ efficient), a total of $4053 e \mu$ candidates are observed, while $4150 \pm 250$ are expected from Standard Model processes. The distribution of the invariant mass $m_{e \mu}$ is presented in Figure 2(a).

Since no evidence for signal is observed the number of events in each search region, as a function of increasing $m_{e \mu}$, are used to set an upper limit on $\sigma\left(p p \rightarrow \tilde{v}_{\tau}\right) \times \operatorname{BR}\left(\tilde{v}_{\tau} \rightarrow e \mu\right)$ using a Bayesian method with a uniform prior for the signal cross section. Figure 2(b) shows the 95\% C.L upper limits on the $\lambda_{311}^{\prime}$ coupling as a function of $m_{\tilde{v}_{\tau}}$ for three values of $\lambda_{312}$. The regions above the curves are excluded in each case. Please refer to [3] for the full limits and exclusion curves on the $\tilde{v}_{\tau}$ production cross section.
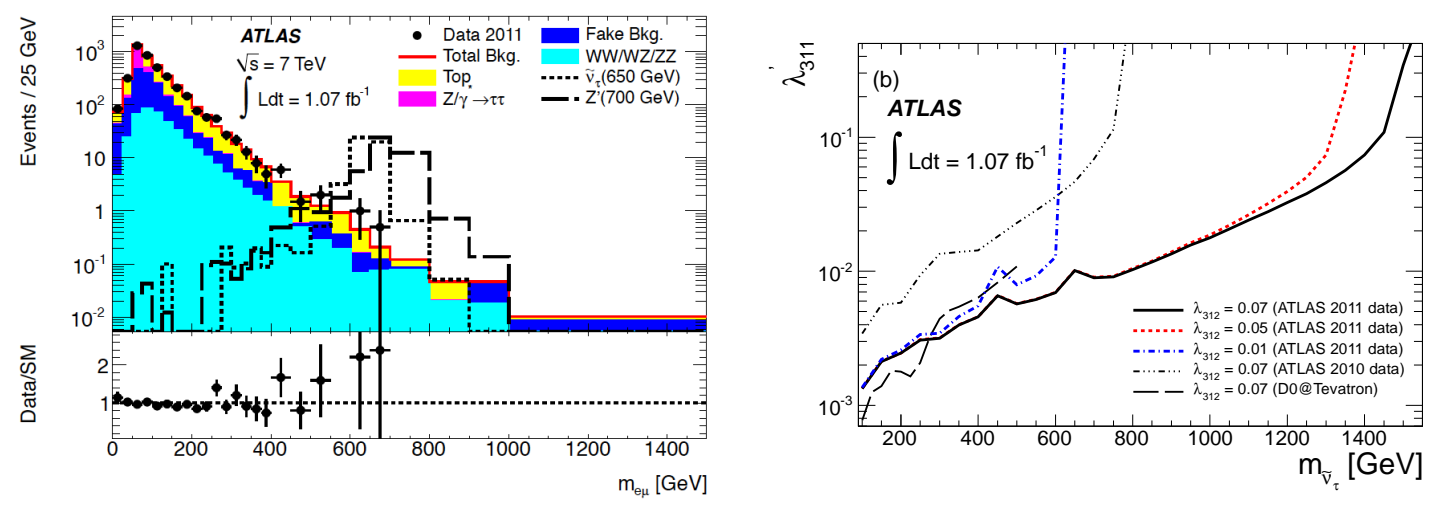

(a) Observed and predicted $e \mu$ invariant mass distributions (b) $95 \%$ C.L. upper limits on the $\lambda_{311}^{\prime}$ coupling as a function of $m_{\tilde{v}_{\tau}}$.

Figure 2: Distribution of events and constraints on the RPV couplings for the $e \mu$ resonance search.

\section{Displaced vertices}

We report on results of a search for a heavy particle decaying into multiple charged particles at a distance of order millimeters to tens of centimeters from the $p p$ interaction point in events containing a muon identified with high $p_{\mathrm{T}}$ [何]. In the SUGRA scenario, such a signature may be manifested via the decay of the lightest supersymmetric particle due to non-zero $\lambda_{2 i j}^{\prime}$ couplings via a diagram such as is shown in Figure 1(b). Current limits on RPV couplings [6] allow for the decay vertex to be displaced and within range of the ATLAS inner tracking detectors.

Events are selected from a data sample of $36 \mathrm{pb}^{-1}$ collected in 2010 and must pass the $p_{\mathrm{T}}>40 \mathrm{GeV}$ single-muon trigger requirement. A primary vertex (PV), originating from the $p p$ collision is required and must contain a minimum of five tracks and a $z$ position within $200 \mathrm{~mm}$. 
Where multiple primary vertices are recorded the one with the highest scalar sum of the $p_{\mathrm{T}}$ of its tracks is used.

We reconstruct a displaced vertex by selecting only tracks with $p_{\mathrm{T}}>1 \mathrm{GeV}$. A large impact parameter $(>2 \mathrm{~mm}$ ), with respect to the transverse position of the $\mathrm{PV}$ is required, rejecting $98 \%$ of all tracks orginiating from the primary $p p$ interaction. A description of the displaced vertex finding algorithm can be found elsewhere [5].

We use the mass of the reconstructed vertex $\left(m_{\mathrm{DV}}\right)$ and the number of reconstructed charged tracks $\left(N_{\mathrm{DV}}^{\mathrm{trk}}\right)$ as discriminating variables for the search. Figure 3 shows the distribution of $m_{\mathrm{DV}}$ vs. $N_{\mathrm{DV}}^{\mathrm{trk}}$ for the selected vertices in the data sample, including vertices that fail the requirements on $m_{\mathrm{DV}}$ and $N_{\mathrm{DV}}^{\mathrm{trk}}$ this is overlaid with a distribution from signal MC. We observe no vertices that satisfy all the selection criteria.

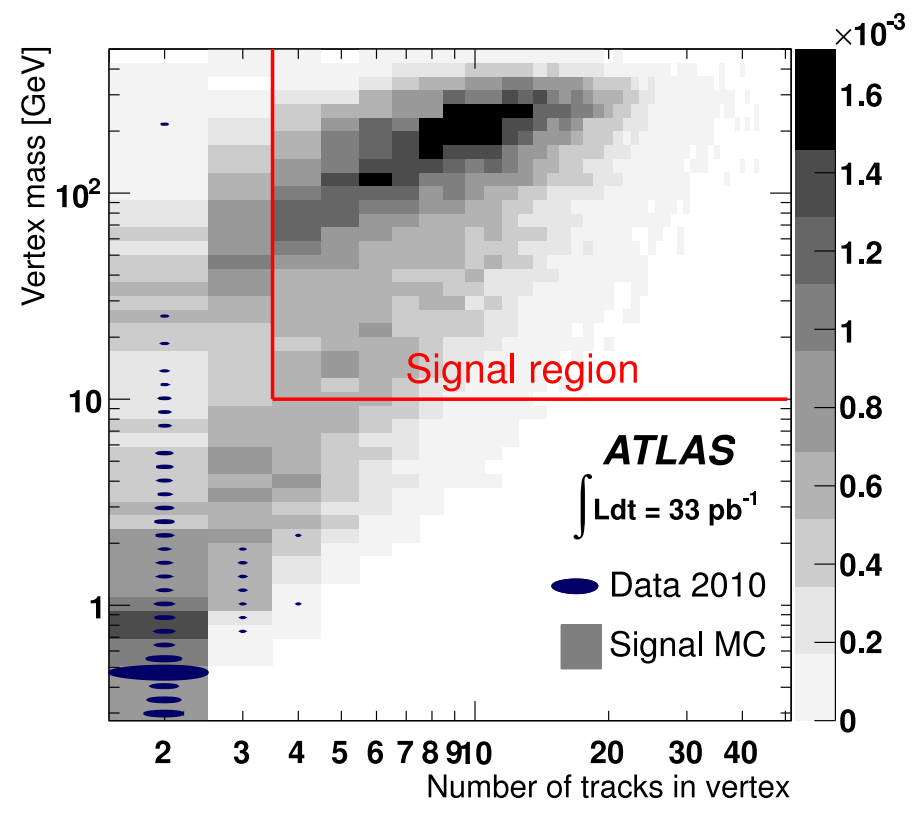

Figure 3: Vertex mass $\left(m_{\mathrm{DV}}\right)$ vs. vertex track multiplicity $\left(N_{\mathrm{DV}}^{\mathrm{trk}}\right)$ for displaced vertices that pass the event selection requirements except the $m_{\mathrm{DV}}$ and $N_{\mathrm{DV}}^{\mathrm{trk}}$ requirements. Shaded bins show the distribution for signal $\mathrm{MC}$, and data are shown as filled ellipses, with the area of the ellipse proportional to the number of events in the corresponding bin. The figure contains 487 data events, of which 251 are in the bin corresponding to $K_{s}^{0}$ decays.

Based on this null observation, we set upper limits on the supersymmetry production crosssection times the branching fraction of the simulated signal decays for different combinations of squark and neutralino masses, and for different values of the product of the speed of light $(c)$ and the neutralino lifetime $(\tau)$. These constraints can be seen in Figure 4 .

\section{Summary}

The ATLAS collaboration has begun mining the data collected during the first two years of $7 \mathrm{TeV}$ operations at the CERN Large Hadron Collider. Novel analyses have already reached ma- 


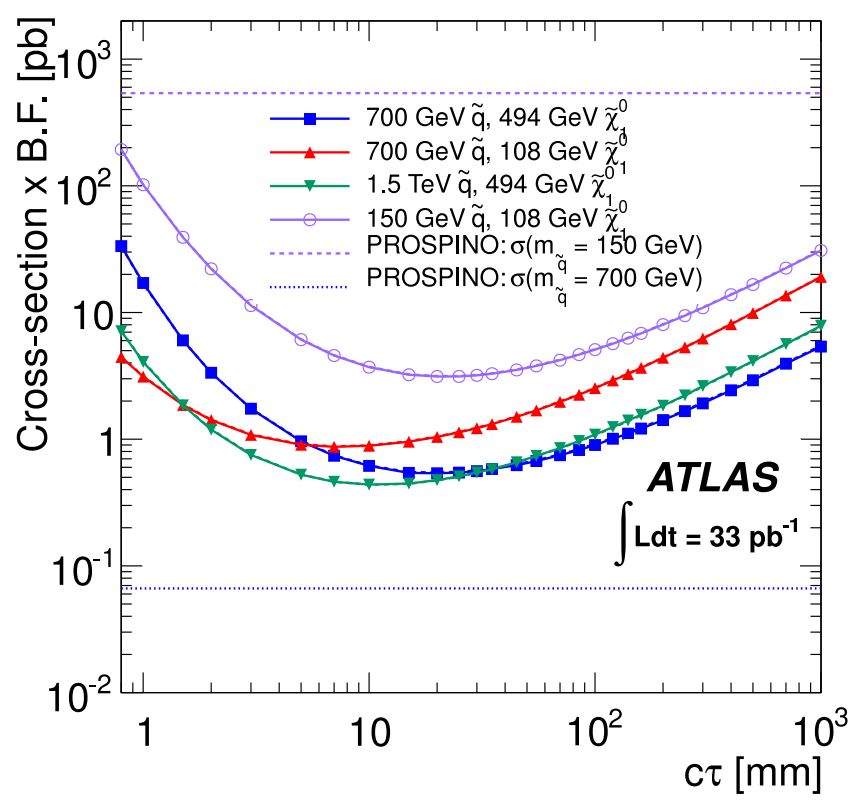

Figure 4: Upper limits at 95\% CL on the production cross-section times branching fraction vs. the neutralino lifetime times the speed of light for different combinations of squark and neutralino masses, based on the observation of zero events in a $33 \mathrm{pb}^{-1}$ data sample. The horizontal lines show the cross-sections calculated from PROSPINO [7] for squark masses of $700 \mathrm{GeV}$ and $150 \mathrm{GeV}$.

turity, and searches for SUSY with R-parity violation with two different approaches have been presented herein. At time of writing no deviations from the Standard Model have been observed in these channels. However, these early analyses will form the foundation of further searches to be performed in the near future.

\section{References}

[1] The ATLAS Collaboration, JINST 3, S08003 (2008).

[2] For some early works on R-violating supersymmetry, see, e.g., L. Hall and M. Suzuki, Nucl. Phys. B 231, 419 (1984); J. Ellis et al., Phys. Lett. B 150, 142 (1985); G. Ross and J. Valle, Phys. Lett. B 151, 375 (1985); S. Dawson, Nucl. Phys. B 261, 297 (1985); R. Barbieri and A. Masiero, Nucl. Phys. B 267, 679 (1986); H. Dreiner and G.G. Ross, Nucl. Phys. B 365, 597 (1991); J. Butterworth and H. Dreiner, Nucl. Phys. B 397, 3 (1993).

[3] The ATLAS Collaboration, CERN-PH-EP-2011-140 [arXiv:1109.3089], accepted by Eur. Phys. J.

[4] The ATLAS Collaboration, Eur. Phys. J. C 70, (2010), 823.

[5] The ATLAS Collaboration, CERN-PH-EP-2011-131 [arXiv:1109.2242], submitted to Phys. Lett. B.

[6] B. C. Allanach et al., Phys. Rev. D 75, 035002 (2007) [arXiv:hep-ph/0609263].

[7] W. Beenakker et al., arXiv:hep-ph/9611232v1. 